\title{
Patient-Reported Outcomes and Experiences with Population Genetic Testing Offered Through a Primary Care Network
}

\author{
Amy A. Lemke, Laura M. Amendola, ${ }^{2}$ Jennifer Thompson, ${ }^{1}$ Henry M. Dunnenberger, \\ Kristine Kuchta, ${ }^{3}$ Chi Wang, ${ }^{3}$ Kristen Dilzell-Yu, ${ }^{1}$ and Peter J. Hulick ${ }^{1}$
}

Aims: To explore patient experiences in a large-scale primary care-based, preemptive genetic testing program. Methods: Patients who received genetic results from the initiative were invited to participate in an online survey 3 weeks postresult disclosure. A 6-month follow-up survey was sent to assess changes over time.

Results: The initial survey was completed by 1646 patients, with 544 completing the 6-month follow-up survey. The following outcomes were high overall: patient-reported understanding of results (cancer: 87\%; cardiac: 86\%); perceived utility (75\%); positive emotions (relieved: 66.8\%; happy: 62.0\%); family result sharing (67.6\%); and satisfaction (87\%), although analysis by demographic factors identified groups who may benefit from additional education and emotional support. Results-related health behaviors and discussions with providers increased over time (screening procedures $6.1 \%$ to $14.2 \% p<0.001$; provider discussion $10.3 \%$ to $25.3 \%$, $p<0.001)$, and were more likely to take place for patients with positive cancer and/or cardiac results (39.8\% vs. $7.6 \%, p<0.001)$. Forty-seven percent of patients reported insurance discrimination concerns, and most $(79.4 \%)$ were not familiar with privacy and nondiscrimination laws. Concerns regarding discrimination and negative emotions decreased between the two survey time points (privacy issues $44.6 \%$ to $35.1 \% p<0.001$; life insurance discrimination concerns $35.5 \%$ to $29.6 \%, p=0.001$; anxiety $8.1 \%$ to $3.3 \%, p<0.001$; and uncertainty $19.8 \%$ to $12.8 \%, p<0.001$ ). These findings led to the development and integration of additional patient resources to improve program implementation.

Conclusion: Our findings highlight patient experiences with and areas of need in a community-based genomic screening pilot initiative using a mixed primary care/genetics provider model to deliver precision medicine.

Keywords: precision medicine, genetic testing, primary care, clinical implementation, patient reported outcomes

\section{Introduction}

$\mathbf{P}$ RECISION MEDICINE AIMS to provide individualized health information to increase awareness of disease risk and medication safety and to enable disease prevention and early detection. Access to personalized health-related genetic information is increasingly available to patients and consumers. This increase in demand for genetic test interpretation and management of genetic risk has led to the development of alternative models of service delivery. Guidance has been proposed to support genetic testing consent and return of genetic results by nongenetics professionals (Faucett et al., 2019; Ormond et al., 2019) and trials incorporating genome sequencing into primary care practice suggest nongenetics pro- viders may manage results appropriately (Vassy et al., 2018). Research exploring a 'genomics first' model returning genetic results to participants in large research cohorts and biorepositories has identified challenges with the integration of such results into clinical workflows and the electronic health record (EHR) (Kullo et al., 2014; Schwartz et al., 2018).

In the past several years, genomics screening programs, which provide results to healthy adults, have been implemented in the context of academic research, provider education, and commercial laboratories (Green et al., 2016; Linderman et al., 2016; Butterfield et al., 2019; Wiesner et al., 2020). The need to implement such programs equitably and with careful assessment of disease penetrance, health outcomes, costs, and patient experiences has been

\footnotetext{
${ }^{1}$ Neaman Center for Personalized Medicine, NorthShore University HealthSystem, Evanston, Illinois, USA.

${ }^{2}$ Division of Medical Genetics, University of Washington, Seattle, Washington, USA.

${ }^{3}$ Center for Biomedical Research Informatics, NorthShore University HealthSystem, Evanston, Illinois, USA.
}

(C) Amy A. Lemke et al., 2021; Published by Mary Ann Liebert, Inc. This Open Access article is distributed under the terms of the Creative Commons Attribution Noncommercial License (http://creativecommons.org/licenses/by-nc/4.0/) which permits any noncommercial use, distribution, and reproduction in any medium, provided the original author(s) and the source are cited. 
emphasized (Murray et al., 2018). Early outcomes from these programs and others have identified patient privacy concerns, incorrect, or incomplete recollection of results and unmet expectations, as well as favorable patient reactions (enthusiasm about the experience, value in health results, relief, low testrelated distress) with minimal reported changes to medical care or lifestyle (Sanderson et al., 2017; Butterfield et al., 2019; Rego et al., 2019; Zoltick et al., 2019; Waltz et al., 2020).

\section{DNA-10K clinical pilot program}

In an effort to integrate genomics into patient care across our health system and enable better disease prevention and early detection, NorthShore University HealthSystem (NorthShore) launched a primary care-based, preemptive genomic screening pilot program in 2018. The initiative provided genetic information to adult participants, irrespective of personal or family history.

First, a prepilot (DNA-1K) with hereditary cancer testing was conducted at four NorthShore primary care sites to assess program feasibility and early patient outcomes (Lemke et al., 2019). Informed by the DNA-1K experience, we implemented a clinical pilot program (DNA-10K) at 14 NorthShore primary care practices beginning in April 2019. Patients were offered no-cost genetic testing for 60 genes associated with hereditary cancer and cardiac conditions, pharmacogenomics (PGx) testing, ancestry, and common trait information through Color ${ }^{\mathrm{TM}}$. Complimentary testing was provided to over 10,000 adult patients through their primary care physician (PCP) in conjunction with their annual examination. The patient workflow is shown in Figure 1. In brief, patients interested in testing consented electronically and the genetic test order was placed by their PCP. Clinical results were available through a patient portal and integrated into the patient's EHR. Genetic counseling through Color ${ }^{\mathrm{TM}}$ was available for all patients and was required for those identified to have a pathogenic variant. Follow-up medical services were provided through NorthShore.

Although research-based testing programs are providing initial insights, information is lacking about patient experiences in receiving and utilizing predictive testing through routine community-based primary care. Examining patient reported outcomes in clinical, primary care-based population genetic testing programs is necessary to successfully deliver this service. Therefore, we conducted a patient survey study with two time points to better understand patient experiences in our genetic testing pilot program, the DNA-10K.

\section{Materials and Methods}

\section{Survey development and measures}

A focus group of seven patients, who received either positive or negative results from the DNA-1K prepilot, was convened in February 2019 to assess patient experiences. Findings from this focus group helped inform question inclusion and categorical responses used in a 48-item, 3-week post-DNA-10K results survey tool (Supplementary File 1). The survey was also informed by multidisciplinary team review, and from five cognitive interviews of NorthShore patients similar to the study participants to increase readability and validity of the tool (Willis, 2005). The Tailored Survey Design method was used as a general guide in survey development and format (Dillman et al., 2014).

Survey domains consisted of decision to have genetic testing, the testing experience, views toward the return of results process, sharing results, feelings and actions after receiving

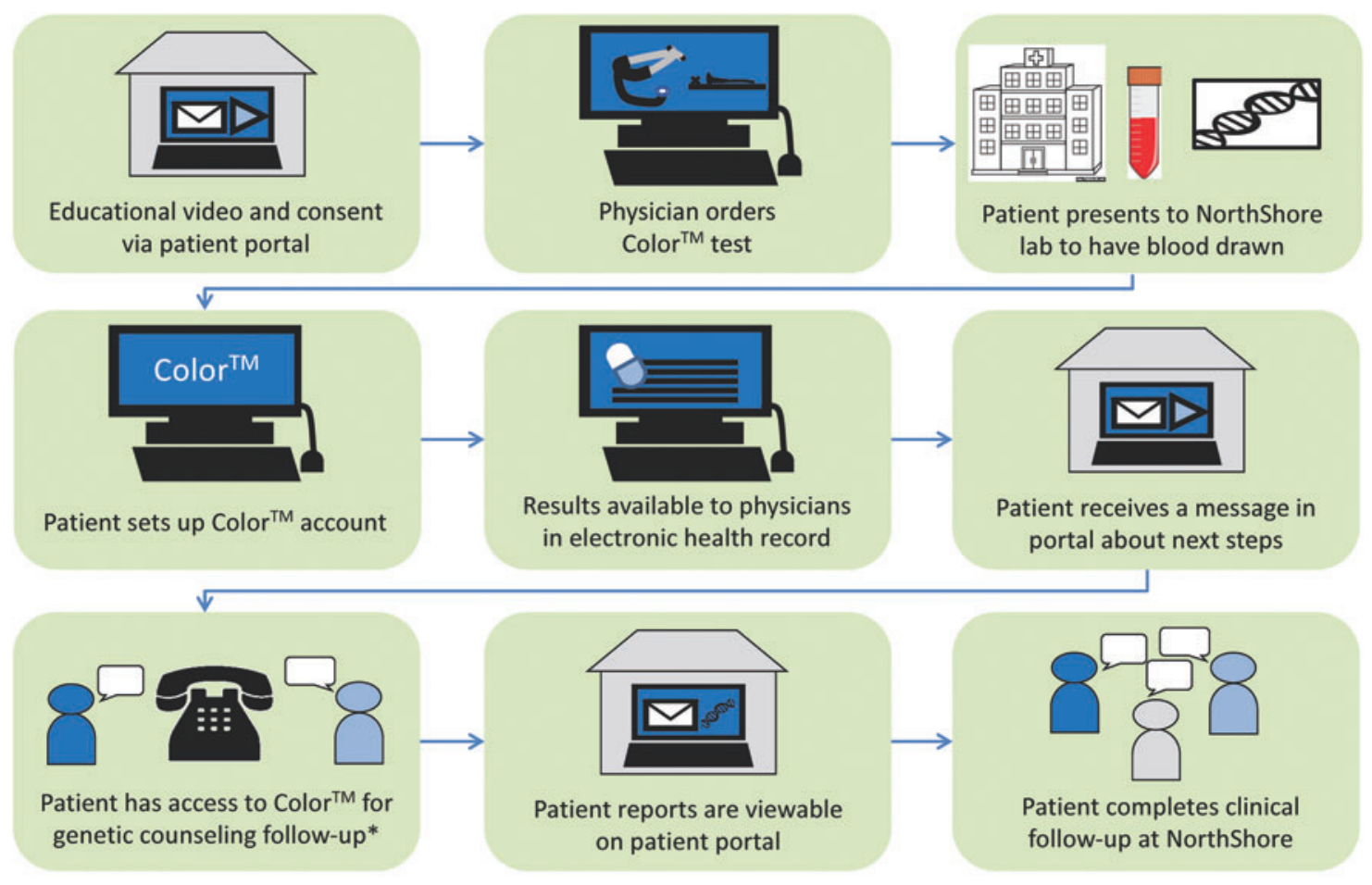

- Patients with a positive cancer/cardiac genetic test result are required to speak with a Color ${ }^{\top M}$ genetic counselor to receive their results.

FIG. 1. DNA-10K patient workflow. Color images are available online. 
results, value of results, privacy and confidentiality, and demographics. Four- or five-point Likert scales were used in most of the response categories for questions assessing participant opinions. Categorical responses were used in other question types and one open-ended question was offered at the end of the survey. A number of survey questions were utilized or adapted from previously published work (Lemke et al., 2018; Li et al., 2018; Zoltick et al., 2019; Cella et al., 2002). The survey was administered using REDCap, an online software program designed to collect and manage survey data (Harris et al., 2009, 2019). To test technical ease of online survey use and length of time to complete, user testing was performed with six individuals. A second follow-up survey was sent 6 months after the initial survey, and included 32 questions from the initial survey to capture changes over time (noted in Supplementary File 1). This study was approved by the NorthShore Institutional Review Board (EH19-080).

\section{Survey recruitment}

Individuals were eligible to participate in the postresult surveys if they were NorthShore patients, 18 years of age and older, and received results from the DNA-10K. An email invitation with a unique participant link for an online survey was sent to potential participants $\sim 3$ weeks after they received their results. Two reminder emails were sent at 1-week intervals. Recruitment for the initial survey occurred from July 2019 through January 2020. Individuals who completed the first survey were sent a second unique participant link 6 months later for a follow-up survey, recruitment for which occurred from January 2020 to May 2020. Participants received a \$5 Starbucks gift card for each survey completed.

\section{Data analysis}

Data were downloaded from REDCap and analyzed using SAS 9.3 (SAS Institute, Inc, 2011). Descriptive statistics were used to summarize participant responses. For questions using the four- or five-point Likert scale response, data were first summarized and reviewed in their original form and then collapsed to two or three categories, respectively, to facilitate analysis and interpretation. Because participants were allowed to skip individual items, the sample size varied by question. Throughout the article, percentages reported reflect the valid percent (excludes missing answers). To compare findings among different demographic factors and genetic result groups (positive and negative), chi-square tests were performed. McNemar's test was conducted to compare responses to questions asked in both the initial and follow-up surveys. All tests were two-tailed and a $p$-value of $\leq 0.05$ was considered statistically significant. Only select comparisons that were statistically significant and clinically meaningful are discussed in the results.

\section{Results}

\section{Response rates}

A total of 1646 out of 6891 invited patients participated in the initial survey (23.9\% response rate). Nine hundred sixtytwo invitations were sent for the 6-month follow-up survey, and 544 completed responses were used for analysis $(56.5 \%$ response rate).

\section{Participant characteristics}

Participants who answered the initial survey were primarily female $(70.5 \%)$ and white $(89.0 \%)$, with a bachelor's degree or higher $(81.4 \%)$ (Table 1). Positive cancer and/or cardiac risk results were reported by $123(7.5 \%)$ participants (Table 2). Participants who answered the 6-month survey were also primarily female $(72.3 \%)$, white $(87.8 \%)$, and had a bachelor's degree or higher $(82.3 \%)$. Fifty-one $(9.4 \%)$ participants who completed the follow-up survey reported a positive cancer and/or cardiac risk result.

\section{Findings from initial survey 3 weeks postresult disclosure}

Motivations, pretest experience, and perceptions of results. When asked to indicate the degree to which several factors impacted their decision to have genetic testing, participants most frequently reported that the desire to learn about their disease risk (73.9\%) and personal curiosity (70.0\%) motivated their decision to a great extent (Fig. 2). Survey participants were also asked to indicate how confident their provider appeared while explaining the risks and benefits of testing: $54.8 \%$ felt that their provider seemed confident. In terms of result comprehension, the majority of patients felt that they had a clear understanding of the results they received (Fig. 3). Overall, patients reported high levels of

Table 1. Initial Survey PARTICIPANT CHARACTERISTICS

\begin{tabular}{|c|c|}
\hline Variables & $\mathrm{n}(\%)$ \\
\hline \multicolumn{2}{|l|}{ Gender $(n=1633)$} \\
\hline Female & $1152(70.5)$ \\
\hline Male & $480(29.4)$ \\
\hline Nonbinary & $1(0.1)$ \\
\hline \multicolumn{2}{|l|}{ Age $(n=1635)$} \\
\hline $18-29$ & $158(9.7)$ \\
\hline $30-39$ & $284(17.4)$ \\
\hline $40-49$ & $352(21.5)$ \\
\hline $50-59$ & $392(24.0)$ \\
\hline $60-69$ & $335(20.5)$ \\
\hline 70 or older & $114(7.0)$ \\
\hline \multicolumn{2}{|l|}{ Race $(n=1621)$ (more than one answer allowed) } \\
\hline White & $1443(89.0)$ \\
\hline Asian & $141(8.7)$ \\
\hline Black or African American & $49(3.0)$ \\
\hline Native Hawaiian or other Pacific Islander & $5(0.3)$ \\
\hline \multicolumn{2}{|l|}{ Ethnicity $(n=1626)$} \\
\hline Hispanic or Latino & $85(5.2)$ \\
\hline Not Hispanic or Latino & $1541(94.8)$ \\
\hline \multicolumn{2}{|l|}{ Highest level of education $(n=1633)$} \\
\hline Less than bachelor's degree & 304 (18.6) \\
\hline Bachelor's degree or higher & $1329(81.4)$ \\
\hline \multicolumn{2}{|l|}{ Household income $(n=1537)$} \\
\hline$<\$ 100,000$ & $546(35.5)$ \\
\hline$\geq \$ 100,000$ & $991(64.5)$ \\
\hline \multicolumn{2}{|l|}{ Health rating $(n=1637)$} \\
\hline Excellent & $314(19.2)$ \\
\hline Very good & $740(45.2)$ \\
\hline Good & $468(28.6)$ \\
\hline Fair & $99(6.0)$ \\
\hline Poor & $16(1.0)$ \\
\hline
\end{tabular}


Table 2. Participants' Experience with Genetics

\begin{tabular}{lc}
\hline Variable & $\mathrm{n}(\%)$ \\
\hline Known genetic condition in patient and/or family $(n=1636)$ \\
Yes & $405(24.8)$ \\
No & $813(49.7)$ \\
Don't know & $418(25.6)$ \\
Previous genetic testing in self $(n=1638)$ & \\
Yes & $281(17.2)$ \\
No & $1332(81.3)$ \\
Don't know & $25(1.5)$ \\
Previous genetic testing in family $(n=1637)$ \\
Yes \\
No & $334(20.4)$ \\
Don't know & $996(60.8)$ \\
Patient reported DNA-10K genetic testing results & \\
( $n=1645)$ & $113(6.9)$ \\
Positive-cancer risk & $22(1.3)$ \\
Positive-cardiac risk &
\end{tabular}

\footnotetext{
${ }^{\mathrm{a}}$ Twelve patients reported both a positive cancer and cardiac risk result
}

satisfaction $(86.9 \%)$ and personal utility (Fig. 4) with the DNA-10K. Higher satisfaction was observed in participants who self-reported to have excellent/very good/good health $(87.9 \%$ vs. $74.8 \%, p=0.003)$ and participants who were $<50$ years old $(89.4 \%$ vs. $84.7 \%, p=0.008)$.
Emotions related to receiving results. Participants were asked how often they experienced various emotions related to their genetic test results over the past week. Most participants reported either sometimes or often feeling relieved $(66.8 \%)$ and happy $(62.0 \%)$, and a minority reported feeling uncertain (22.6\%), anxious/nervous (8.2\%), disappointed (6.3\%), upset $(2.8 \%)$, and regret (1.7\%). Participants with positive cancer and/or cardiac results were more likely to feel negative emotions (uncertain: $p=0.008$; anxious/nervous: $p<0.001$; upset: $p<0.001$; regret: $p=0.001$; and disappointment: $p<0.001$ ) and participants with negative cancer and/or cardiac results were more likely to report positive emotions $(p<0.001$ for happy and relieved). In addition, participants who reported themselves to be in fair/poor health, who do not identify as white, and who had an income of $<\$ 100 \mathrm{~K}$ were more likely to report negative emotions, such as feeling anxious/nervous and uncertain (health: $p<0.001$ for each; not white: $p \leq 0.011$ for each; income: $p<0.001$ for each).

Posttest plans and actions. At 3 weeks postresult receipt, few participants had spoken with their provider $(10 \%)$ or the Color genetic counselor $(9.1 \%)$ about their results, although more $(44.4 \%$ and $14.5 \%$, respectively) reported that they planned to. Participants were more likely to have spoken with their provider if they reported a positive cancer and/or cardiac risk result $(39.8 \%$ vs. $7.6 \%, p<0.001)$, were $<50$ years old $(11.7 \%$ vs. $8.6 \%, p=0.037)$, or reported a

\section{To what extent did the following factors play a part in your decision to be tested?}

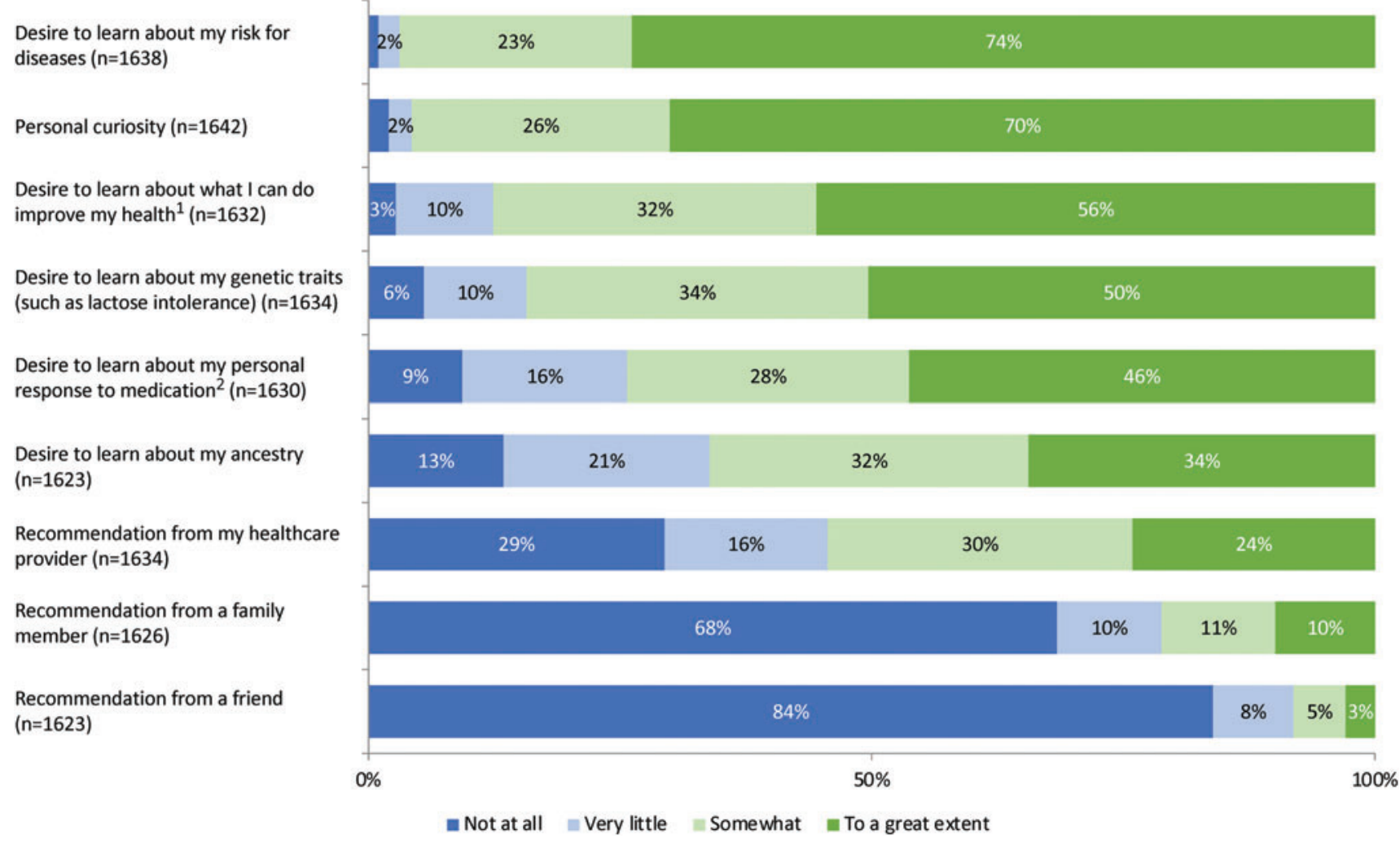

FIG. 2. (1) Women were more likely to report that the desire to learn about ways to improve health influenced their decision to a great extent $(59.0 \%$ vs. $47.3 \%, p<0.001)$. (2) Participants $\geq 50$ years old were interested in their personal response to medications to a great extent more often than participants $<50$ years old $(51.1 \%$ vs. $41.9 \%, p<0.001)$. Color images are available online. 


\section{I have a clear understanding of my test results from the DNA-10K}

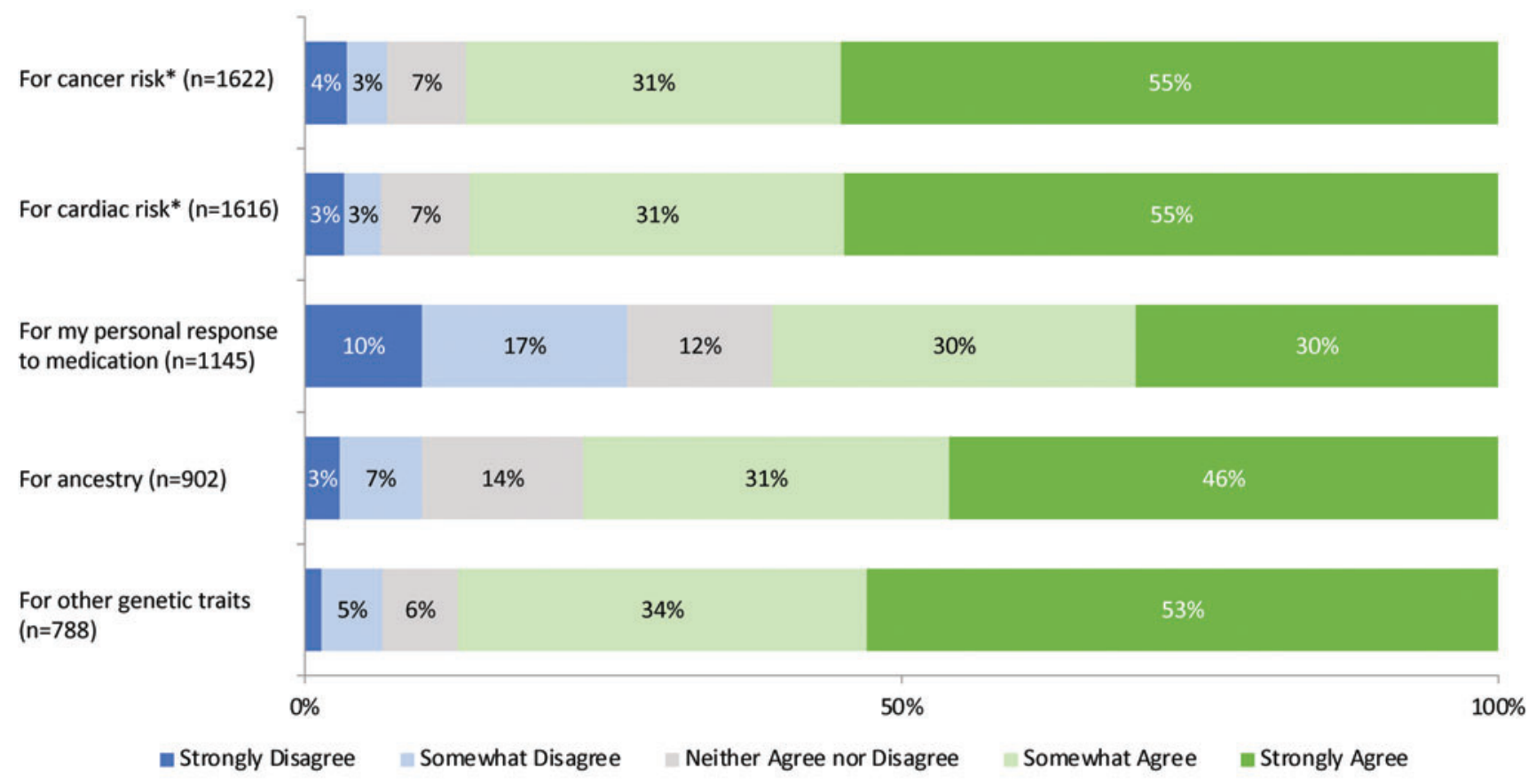

*Statistically Significant $(\mathrm{P} \leq 0.025)$

FIG. 3. Participants were less likely to report clear understanding of cancer or cardiac risk if they reported themselves to be in fair/poor health (cancer: $77.2 \%$ vs. $87.3 \%, p=0.004$; cardiac: $87.2 \%$ vs. $73.7 \%, p<0.001$ ), did not identify as white (cancer: $81.4 \%$ vs. $87.5 \%, p=0.006$; cardiac: $87.0 \%$ vs. $81.3 \%, p=0.025$ ), or had less than a bachelor's degree (cancer: $80.1 \%$ vs. $88.1 \%, p=0.004$; cardiac: $87.7 \%$ vs. $79.7 \%, p=0.003)$. Color images are available online.

family history of a genetic condition $(14.4 \%$ vs. $9.1 \%$, $p=0.011)$. Of the participants who reported talking with a Color $^{\mathrm{TM}}$ genetic counselor $(n=150)$, approximately half $(52.0 \%)$ either somewhat or strongly agreed that it was easy to follow-up on the recommendations through NorthShore, while $20.7 \%$ indicated that this was not applicable to them.

The most common actions taken by participants 3 weeks after receiving results were to have a screening procedure $(7.3 \%)$ and to schedule an appointment with a specialist (4.1\%). Participants with a positive cancer and/or cardiac result were more likely than those with a negative result to have taken these two actions (screening procedure: $19.7 \%$ vs. $6.3 \%, p<0.001$; appointment: $32.5 \%$ vs. $1.8 \%, p<0.001$ ). Few participants reported making changes to their medications $(1.6 \%)$, insurance coverage $(1.0 \%)$, and advanced planning $(1.3 \%)$.

Family communication. Participants were asked a series of family communication-related questions. Most participants either had $(67.6 \%)$ or planned to $(9.7 \%)$ discuss results with family members, and participants with a positive cancer and/or cardiac result were more likely to have done so $(91.1 \%$ vs. $65.7 \%, p<0.001)$. Some participants $(21.3 \%)$ wanted additional resources to help communicate results with family members. Participants were more likely to desire these resources if they did not identify as white $(p=0.018)$, were $\geq 50$ $(p<0.001)$, had less than a bachelor's degree $(p=0.026)$, and an income $<\$ 100 \mathrm{~K}(p=0.001)$. At the time of the initial survey, $19.3 \%$ of participants reported that one or more fam- ily members had pursued genetic testing after learning the participant's results, and those with a positive cancer and/or cardiac result were more likely to report this $(34.8 \%$ vs. $17.5 \%, p<0.001)$.

Privacy and confidentiality. Approximately half (47.0\%) of participants reported that they were concerned somewhat or to a great extent about the privacy of their genetic test results. Regarding health and life insurance, $38.3 \%$ and $36.4 \%$, respectively, indicated concern about potential discrimination based on their results. Individuals who did not identify as white were more likely to report concern about privacy of test results $(57.9 \%$ vs. $45.7 \%, p<0.001)$, health insurance discrimination $(50.6 \%$ vs. $36.6 \%, p=0.005)$, and life insurance discrimination $(45.5 \%$ vs. $35.0 \%, p=0.012)$. The majority of patients $(79.4 \%)$ reported lack of familiarity with the Genetic Information Nondiscrimination Act (GINA).

\section{Comparison of initial and follow-up surveys}

The responses of patients who completed both the initial and 6-month follow-up surveys were compared and analyzed $(n=544)$. In the 6 months between the first and second survey, an increase in completed follow-up actions was observed. An additional 15\% (increase from $10.3 \%$ to $25.3 \%$ ) of participants discussed their results with their health care provider $(p<0.001)$, and another $8.5 \%(69.3 \%$ to $77.8 \%)$ discussed them with a family member $(p<0.001)$. Participants also scheduled more appointments with specialists 


\section{Personal Utility}

I found my genetic test results to be helpful to me in my health care decision-making at this $\operatorname{time}^{1}(n=1642)$

I think my genetic test results will be helpful to me in my health care decision-making for the future ${ }^{2}(n=1639)$

My genetic test results helped me get a better understanding of my health status ${ }^{2}(n=1641)$

After receiving my genetic test results I feel more confident in managing my health care $^{1}(n=1644)$

After receiving my genetic test results I am more likely to follow screening recommendations from my health care provider $^{1}(n=1643)$

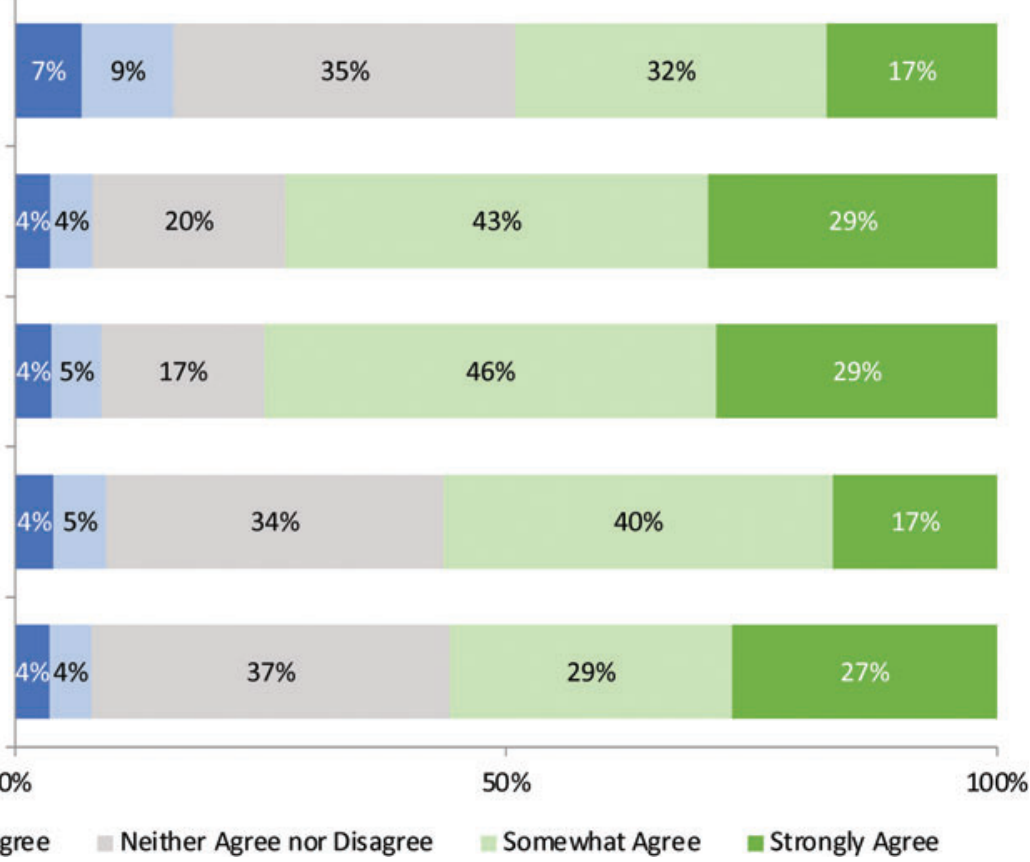

=Strongly Disagree $\quad$ Somewhat Disagree $\quad$ Neither Agree nor Disagree $\quad$ Somewhat Agree $\backsim$ Strongly Agree

FIG. 4. (1) Those with positive cancer and/or cardiac results were more likely to agree that they felt more confident managing their health care, were more likely to follow recommendations from their health care provider, and that results were helpful for current decision making (confident: $68.6 \%$ vs. 55.4\%, $p<0.001$; recommendations: $73.8 \%$ vs. $54.4 \%$, $p<0.001$; decision making: $61.8 \%$ vs. $48.0 \%, p<0.001)$. (2) Participants who self-reported to have excellent/very good/good health were more likely to agree that their genetic test results helped them better understand their health status $(76.1 \%$ vs. $56.5 \%, p<0.001)$ and will help them make health care decisions in the future $(73.6 \%$ vs. 57.0\%, $p=0.005)$. Color images are available online.

during this time $(2.8 \%$ to $6.6 \%, p<0.001)$ and increased their utilization of screening procedures such as mammograms, from $6.1 \%$ to $14.2 \%(p<0.001)$.

With regard to participant feelings about their genetic test results, a decrease in negative emotions was observed between the initial and 6-month survey (Fig. 5). Most notably, the percentage of patients who reported feeling anxious about their test results decreased from $8.1 \%$ to $3.3 \%(p<0.001)$. Uncertainty about results was also reduced during this time period from $19.8 \%$ to $12.8 \%(p<0.001)$. Feelings of regret and disappointment remained constant over time $(0.6 \%$ to $1.3 \% ; p=0.392$ and $4.6 \%$ to $4.6 \% ; p=0.249$, respectively), and overall satisfaction with the decision to have testing also did not change $(93.4 \%$ to $90.0 \%, p=0.181)$. Finally, patient

$50 \%$

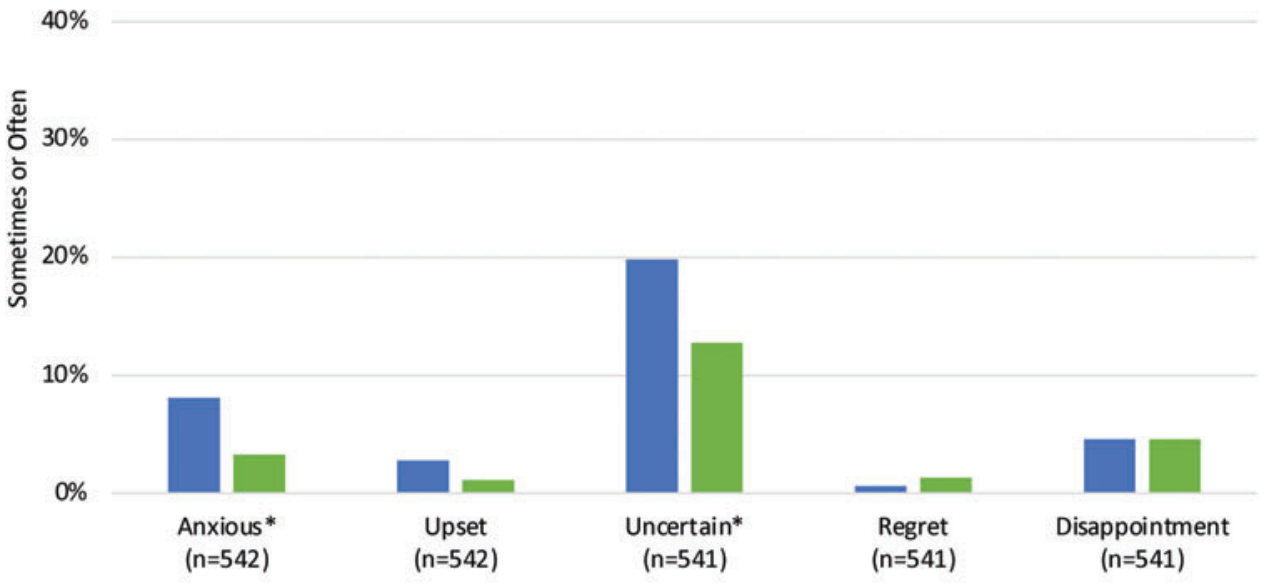

FIG. 5. Patient feelings about their genetic test results at 3 weeks and at 6 months. Color images are available online. 
concerns about privacy and life insurance discrimination related to their genetic test results decreased over the followup period, while health insurance discrimination concerns did not change significantly (Fig. 6).

\section{Discussion}

This work presents patient-reported outcomes from a large-scale clinical genetic testing pilot program integrated within primary care. Patients receiving results through the DNA-10K who participated in postresults surveys reported overall high levels of satisfaction, understanding, personal utility, positive emotions, and family communication. Privacy and confidentiality concerns were reported by about half of survey participants, and the majority indicated limited knowledge of GINA. Concerns about genetic discrimination and negative emotions related to results decreased over time. In addition, actions taken 3 weeks after results disclosure were understandably limited, but were appropriately higher in those with positive results, and increased overall at the 6-month follow-up survey.

Analysis of survey responses by demographic factors identified groups that may benefit from additional or more tailored resources at various points throughout the program. For example, perceived understanding across result types was high overall; however, participants with lower education, lower income, or who did not identify as white reported feeling less confident in their comprehension and were more likely to indicate that they wanted additional resources to share results with family. In response to this finding, we are in the process of developing additional education materials for our program in multiple languages and making other program modifications to increase patient understanding and facilitate follow-up (Lemke et al., 2020). All patients are likely to benefit from the provision of resources that support patient education, family communication, and emotional responses. Further research is needed to explore ways to identify individuals who are more likely to have lower result understanding and negative emotional reactions, and how best to provide additional pre- and posttest support when these needs are identified.

While the potential for negative psychological outcomes in patients having genetic tests has been reported in the literature (Weiner, 2014), the present study did not identify significant distress in DNA-10K survey participants, and initial negative emotions decreased over time. These findings are consistent with reported emotions for participants enrolled in genomic implementation research studies (Robinson et al., 2019; Zoltick et al., 2019). Combined with our findings, this suggests that large-scale genomic sequencing programs can be implemented in a primary care setting at an institutional level without a high level of concern for patient emotional burden.

Apprehension over privacy and discrimination have also been reported in the literature as a concern for patients considering and enrolled in genomic sequencing studies (Gilmore et al., 2017; Amendola et al., 2018; Zoltick et al., 2019). A lack of familiarity in the United States general population with GINA and its protections has also been reported (Green et al., 2015). Consistent with this research,

Privacy of results* $(n=541)$

Health Insurance $(n=541)$

Life Insurance* $(n=540)$

Knowledge of GINA*

( $n=542)$
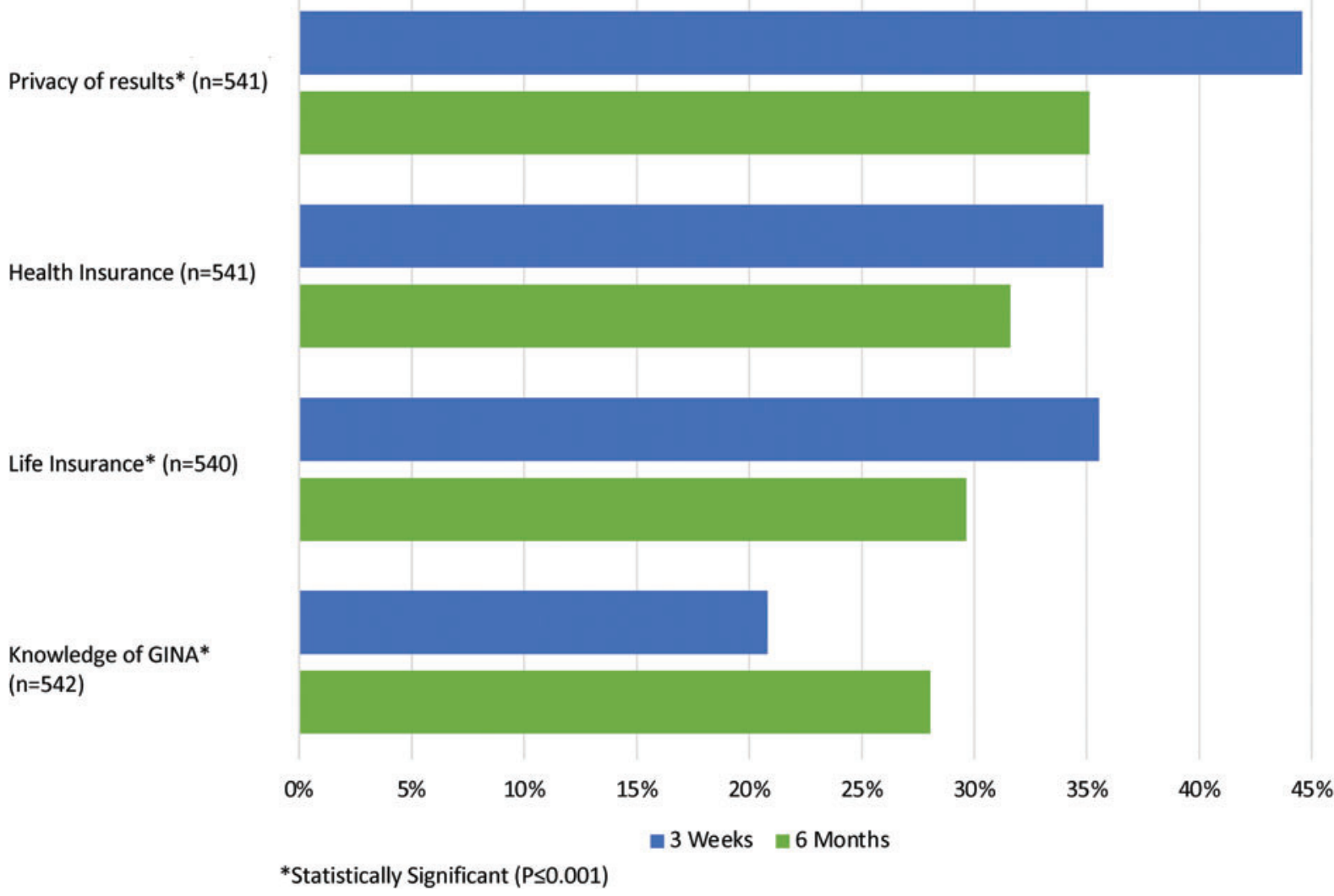

FIG. 6. Patient privacy and discrimination concerns and knowledge of the Genetic Information Nondiscrimination Act at 3 weeks and 6 months. Color images are available online. 
approximately half of our participants reported concerns about insurance discrimination and the majority were not familiar with GINA, although related content was included in the consent information provided to patients. Privacy and discrimination concerns decreased over time, which is also consistent with reports in the literature, although in a different testing context (Niu et al., 2019). These findings suggest that additional patient education on these topics may be needed at various points throughout the testing program. To address this issue, we developed a GINA education video to supplement consent information for the DNA-10K.

With regard to patient actions after results receipt, the number of participants who discussed their results with their provider and took action increased over the 6-month time period. Approximately $20 \%$ of survey participants reported follow-up actions, which is similar to reports from previous research (Sanderson et al., 2017; Butterfield et al., 2019; Zoltick et al., 2019). It is possible that for most participants, no additional or immediate follow-up recommendations were necessary, especially in participants with negative results or those with a positive result related to a future risk, which is more likely to occur in a preemptive genetic screening program. Approaches to better assess the effects of genomic sequencing on downstream health care utilization have been suggested (Mackay et al., 2020), but barriers to patient follow-up still require evaluation to identify tools to successfully increase patient actions and improve outcomes across broad populations in these types of programs. To ensure that patients are getting appropriate follow-up care, clinical care pathways to systematically guide and monitor patient actions are being developed in the NorthShore EHR. In addition, we are piloting a genetic care coordinator role to assist patients and PCPs with appropriate medical management and to transition patients from primary to specialty care (Lemke et al., 2020).

The inclusion of nongenetics providers in a genomics care model is one potential method by which to scale genetic services. However, approximately half of survey participants did not feel that their PCPs were confident explaining their DNA-10K results. Nongenetic provider education is an established area for additional research and attention for the field of clinical genetics. It has been suggested that providers need additional resources and systems-level support to access relevant information at the point of care (Hamilton et al., 2017; Wilkes et al., 2017). To assess experiences and needs of the PCPs in our health system participating in the DNA-10K, we conducted a mixed-methods study, which has informed the development of resources to support PCPs in managing patient genetic results (Lemke et al., 2020).

The generalizability of these findings is limited by several factors. First, the perspectives of the patients who responded to postresults surveys may differ from those who chose not to respond. The majority of patients who participated were women, white, and had higher incomes, thus their responses may not reflect the experience and views of patients in a more diverse health care setting. Furthermore, $\sim 25 \%$ of participants had previous exposure to genetics through personal or familial testing and/or known disease, which may have influenced their information seeking, reactions, and concerns regarding the DNA-10K program. In addition, due to privacy concerns brought up by NorthShore's data governance committee, actual patient genetic test results from the EHR could not be linked to survey responses. Therefore, patients were asked to self-report these results. This introduces the potential for inaccurate patient recall of their genetic results. However, we expect that the number of patients who inaccurately reported their results is likely small given that all patients with a positive result were referred for genetic counseling, the self-reported positivity rate was similar to the known result positivity rate, and the majority of patients reported a clear understanding of their results. While this is a limitation, examining patient feelings and actions based on patient reported results provides a pragmatic exploration of the downstream effects of this type of testing program. Finally, no adjustments were made for multiple comparisons to control Type I error. Some statistically significant findings may be due to the large number of comparisons made in this analysis.

\section{Conclusion}

The successful delivery of a population genetic health initiative program requires collaboration and engagement with key stakeholders such as providers and patients. The results of this study identified areas of need for additional education and assistance, as well as informed the development of solutions to improve the delivery of personalized medicine in primary care. These patient-reported findings from DNA-10K can aid in the establishment and implementation of other large-scale, community-based genetic sequencing programs. Future research is needed to examine diverse patient population perspectives on the delivery of preemptive genomic testing in a variety of health care settings.

\section{Acknowledgments}

The authors acknowledge the contributions of the Color team for their help with program operations and genetic counseling. We especially thank the patients who participated in this study.

\section{Author Disclosure Statement}

No competing financial interests exist.

\section{Funding Information}

This work received support from NorthShore's Transformation through Innovation Fund.

\section{Supplementary Material}

Supplementary File 1

\section{References}

Amendola LM, Robinson JO, Hart R, et al. (2018) Why patients decline genomic sequencing studies: experiences from the CSER consortium. J Genet Couns 27:1220-1227.

Butterfield RM, Evans JP, Rini C, et al. (2019) Returning negative results to individuals in a genomic screening program: lessons learned. Genet Med 21:409-416.

Cella D, Hughes C, Peterman A, et al. (2002) A brief assessment of concerns associated with genetic testing for cancer: the Multidimensional Impact of Cancer Risk Assessment (MICRA) questionnaire. Health Psychol 21:564-572. 
Dillman DA, Smyth JD, Christian LM (2014) Internet, Phone, Mail, and Mixed-Mode Surveys: The Tailored Method, 4th Edition. John Wiley and Sons, Inc., Haboken, New Jersey.

Faucett WA, Peay H, Coughlin CR (2019) Genetic testing: consent and result disclosure for primary care providers. Med Clin North Am 103:967-976.

Gilmore MJ, Schneider J, Davis JV, et al. (2017) Reasons for declining preconception expanded carrier screening using genome sequencing. J Genet Couns 26:971-979.

Green RC, Goddard KAB, Jarvik GP, et al. (2016) Clinical sequencing exploratory research consortium: accelerating evidence-based practice of genomic medicine. Am J Hum Genet 98:1051-1066.

Green RC, Lautenbach D, McGuire AL (2015) GINA, genetic discrimination, and genomic medicine. N Engl J Med 372: 397-399.

Hamilton JG, Abdiwahab E, Edwards HM, et al. (2017) Primary care providers' cancer genetic testing-related knowledge, attitudes, and communication behaviors: a systematic review and research agenda. J Gen Intern Med 32:315-324.

Harris PA, Taylor R, Minor BL, et al. (2019) The REDCap consortium: building an international community of software platform partners. J Biomed Inform 95:103208.

Harris PA, Taylor R, Thielke R, et al. (2009) Research electronic data capture (REDCap) - a metadata-driven methodology and workflow process for providing translational research informatics support. J Biomed Inform 42:377-381.

Kullo IJ, Haddad R, Prows CA, et al. (2014) Return of results in the genomic medicine projects of the eMERGE network. Front Genet 5:50.

Lemke AA, Amendola LM, Kuchta K, et al. (2020) Primary care physician experiences with integrated population-scale genetic testing: a mixed-methods assessment. J Pers Med 10: 165.

Lemke AA, Dunnenberger HM, Thompson J, et al. (2019) Preemptive hereditary cancer genetic testing in primary care: leveraging early program data for process improvement. Available at: https://www.ashg.org/wp-content/uploads/2019/ 10/ASHG-2019-poster-abstracts.pdf. Accessed October 16, 2020.

Lemke AA, Hulick PJ, Wake DT, et al. (2018) Patient perspectives following pharmacogenomics results disclosure in an integrated health system. Pharmacogenomics 19:321-331.

Li M, Bennette CS, Amendola LM, et al. (2018) The feelings about genomiC testing results (FACToR) questionnaire: development and preliminary validation. J Genet Couns 28: 477-490.

Linderman MD, Nielsen DE, Green RC (2016) Personal genome sequencing in ostensibly healthy individuals and the PeopleSeq Consortium. J Pers Med 6:14.

Mackay Z, Dukhovny D, Phillips K, et al. (2020) Quantifying downstream healthcare utilization in studies of genomic testing. Value Health 23:559-565.

Murray MF, Evans JP, Angrist M, et al. (2018) A Proposed Approach for Implementing Genomics-Based Screening Programs for Healthy Adults. NAM Perspectives. Discussion Paper, National Academy of Medicine, Washington, DC.

Niu X, Amendola LM, Hart R, et al. (2019) Clinical exome sequencing vs. usual care for hereditary colorectal cancer diagnosis: a pilot comparative effectiveness study. Contemp Clin Trials 84:105820.
Ormond KE, Hallquist MLG, Buchanan AH, et al. (2019) Developing a conceptual, reproducible, rubric-based approach to consent and result disclosure for genetic testing by clinicians with minimal genetics background. Genet Med 21:727-735.

Rego S, Dagan-Rosenfeld O, Bivona SA, et al. (2019) Much ado about nothing: a qualitative study of the experiences of an average-risk population receiving results of exome sequencing. J Genet Couns 28:428-437.

Robinson JO, Wynn J, Biesecker B, et al. (2019) Psychological outcomes related to exome and genome sequencing result disclosure: a meta-analysis of seven Clinical Sequencing Exploratory Research (CSER) Consortium studies. Genet Med 21:2781-2790.

Sanderson SC, Linderman MD, Suckiel SA, et al. (2017) Psychological and behavioural impact of returning personal results from whole-genome sequencing: the HealthSeq project. Eur J Hum Genet 25:280-292.

SAS Institute Inc. (2011) SI. Base SAS ${ }^{\circledR} 9.3$ Procedures Guide. SAS Institute Inc., Cary, NC.

Schwartz MLB, McCormick CZ, Lazzeri AL, et al. (2018) A model for genome-first care: returning secondary genomic findings to participants and their healthcare providers in a large research cohort. Am J Hum Genet 103:328-337.

Vassy JL, Davis JK, Kirby C, et al. (2018) How primary care providers talk to patients about genome sequencing results: risk, rationale, and recommendation. J Gen Intern Med 33: 877-885.

Waltz M, Meagher KM, Henderson GE, et al. (2020) Assessing the implications of positive genomic screening results. Pers Med 17:101-109.

Weiner C (2014) Anticipate and communicate: ethical management of incidental and secondary findings in the clinical, research, and direct-to-consumer contexts (December 2013 report of the Presidential Commission for the Study of Bioethical Issues). Am J Epidemiol 180:562-564.

Wiesner GL, Rahm AK, Appelbaum P, et al. (2020) Returning results in the genomic era: initial experiences of the eMERGE network. J Pers Med 10:30.

Wilkes MS, Day FC, Fancher TL, et al. (2017) Increasing confidence and changing behaviors in primary care providers engaged in genetic counselling. BMC Med Educ 17:163.

Willis GB (2005) Cognitive Interviewing. A Tool for Improving Questionnaire Design. Sage Publications, Thousand Oaks, California.

Zoltick ES, Linderman MD, McGinniss MA, et al. (2019) Predispositional genome sequencing in healthy adults: design, participant characteristics, and early outcomes of the PeopleSeq Consortium. Genome Med 11:10.

Address correspondence to: Amy A. Lemke, PhD, MS Neaman Center for Personalized Medicine NorthShore University HealthSystem 1001 University Place, Suite 106 Evanston, IL 60201 USA

E-mail: alemke@northshore.org; lemkeaa@gmail.com 